\title{
A New Approach to Characterising Aspheric Surfaces
}

\author{
W. Sun*, J. W. McBride, M. Hill \\ wjs@soton.ac.uk \\ School of Engineering Sciences, University of Southampton, Highfield SO17 1BJ, UK
}

\begin{abstract}
In this paper, a new approach to fitting aspheric surfaces in three-dimensional space is proposed, based on the nonlinear least-squares algorithm. The method is superior to conventional solutions as all the surface parameters can be estimated simultaneously based on the design equation, thus allowing the result to be directly compared to design parameters. Conventionally, aspheric surfaces can be fitted with simplified surface models, such as a second order surface or polynomial model. Using this approach the estimated parameters cannot be compared with the design values, breaking the link between the designed and measured surface. The new method is developed here and tested on computer simulated aspheric surfaces. Both ideal surfaces and surfaces with random irregularities are considered. Issues regarding the application of the fitting method to real measured surfaces are discussed.
\end{abstract}

\section{Keywords}

Aspheric surfaces; fitting algorithm; nonlinear least-squares 


\begin{tabular}{|c|c|}
\hline \multicolumn{2}{|l|}{ Nomenclature } \\
\hline$A_{4} A_{6} A_{8} A_{10} A_{12}$ & polynomial coefficients \\
\hline$c$ & reciprocal of vertex radius $(1 / R)$ \\
\hline$k$ & conic constant \\
\hline$l$ & scanning length \\
\hline$m s$ & \\
\hline$p_{1}, p_{2}, p_{3}, \ldots p_{9}$ & intermediate parameters used in the derivation \\
\hline$a_{1}, a_{2}, a_{3}, \ldots a_{9}$ & \\
\hline$n$ & number of data points \\
\hline$r$ & radial distance from the $\mathrm{Z}$ axis, $r=\sqrt{x^{2}+y^{2}}$ \\
\hline$R$ & vertex radius \\
\hline$(x, y, z)$ & location of a data point on the surface in the XYZ coordinate system \\
\hline $\bar{z}$ & estimated data \\
\hline$c^{\prime} k^{\prime} A_{4}^{\prime} A_{6}^{\prime}$ & aspheric surface parameters with negative designed vertex radius \\
\hline$\Delta c \Delta m \Delta A_{4} \Delta A_{6}$ & change of parameters in iteration process \\
\hline$\Delta l$ & sampling interval \\
\hline$\Delta \boldsymbol{p}_{s}$ & change of parameters with iterations \\
\hline$\delta$ & sum of the parameters' absolute change. $\delta=\sum\left|\Delta \boldsymbol{p}_{s}\right|$ \\
\hline
\end{tabular}

\section{Introduction}

In traditional optical systems, a number of spherical lenses are used in groups to reduce aberrations. By using aspheric lenses, the number of lenses can be significantly reduced compared with a spherical optical system. 
Therefore, an optical system using aspheric lenses has the potential to be smaller than an equivalent optical system with spherical lenses. Additionally it is common for aspheric surfaces to be used to correct spherical departures, coma, astigmatism and distortion, as well as to avoid pupil aberrations [1,2].

Aspheric surfaces are relatively easy to manufacture, but the metrology is difficult. Generally the surface slope and the aspheric departure determine the difficulty of the measurement. The two main issues with respect to metrology are: how to precisely measure aspheric surfaces, particularly in three dimensions and how to characterise the measured surfaces. This paper is mainly focused on the latter, the characterisation of aspheric surfaces.

\subsection{Definition of aspheric surfaces}

An aspheric surface is generally defined as a rotationally symmetrical surface that gradually varies in surface power from the centre towards the edge in a radial fashion [3]. The surface can be represented by parameters based on geometric equations. The general equation for an aspheric surface is given in Equation (1).

$$
z=\frac{c r^{2}}{1+\sqrt{1-(1+k) c^{2} r^{2}}}+A_{4} r^{4}+A_{6} r^{6}+A_{8} r^{8}+A_{10} r^{10}+A_{12} r^{12}
$$

In Equation $1, \frac{c r^{2}}{1+\sqrt{1-(1+k) c^{2} r^{2}}}$ is the conic section; $(x, y, z)$ represent the location of a data point on the surface (see Figure 1) in the XYZ coordinate system 


\subsection{Characterisation of aspheric surfaces}

The aim of surface characterisation is to determine the geometrical form of a measured surface. The phrase

"Geometrical form", can also be known as the "ideal geometrical surface", "design form", or "nominal surface" [4]. In surface characterisation, the geometrical form is usually known or predetermined. The optimised parameters can therefore be estimated in terms of the predetermined geometrical form. Thus, the geometric form, form deformation and the residuals can be observed and the form characterisation results used to compare the measured surface with the designed surface.

There is little published research regarding the fitting process for aspheric surfaces though the issue of the fitting of conic sections has been broadly investigated. Héctor reported a method for the fitting of a conic aspheric curve [5], where a general second order function which accommodates the position and the orientation of a surface is used. The calculation of the parameters of the conic aspheric curve was based on a linear least-squares procedure. A full study of the fitting of conic sections has been presented by Zhang [6], where $2 \mathrm{D}$ conic sections are transformed into a linear problem. Different least-squares fitting techniques were compared and the robustness of the fitting algorithms discussed.

In [7] Gugsa and Davies proposed a method to estimate the conic constant for an aspheric micro lens using scanning white light interferometry. From the measured surface an estimate for the vertex radius is found by varying the size of the sampling area of the measured surface. By varying the value of the conic constant $k$, 
the best-fit profile of the measured surface is achieved using a least-squares minimization method. The vertex radius, the sag of the lens, the lens aperture and lens centre position of curvature are estimated. The biggest issue regarding this method is that it treats the measured surface as a conic surface without consideration to the designed form. In the case of measuring an aspheric surface, the estimation of the conic constant $k$ may be biased by neglecting of polynomial coefficients, referring to Equation 1. Also, the quality of the estimation of the vertex radius is dependent on the quality of the sphere fitting algorithm, where the fitting results may be affected by the surface noise, particularly within small surface segment angles. Furthermore, as the fitting algorithm uses an estimated value of the vertex radius as the true vertex radius, the best-fit parameter $k$ may be biased if the estimation of vertex radius deviates from the true value. A similar method was proposed in [8], where a nonlinear least-squares sphere fitting method was applied to varying surface areas allowing a prediction of the vertex radius. The estimation of other parameters is also based on the estimation of the vertex radius. The method is superior to the one proposed by Gugsa and Davies as it allows an investigation of all parameters based on the design equation. This is referred to as an indirect solution. However, this method and the method suggested by Gugsa and Davies are both limited by the precision of the estimated vertex radius. One critical issue is the precision of the nonlinear least-squares sphere fitting algorithm applied to small segment angle surfaces.

The reliability of the nonlinear least-squares sphere fitting algorithm has been investigated in [9], where two essential aspects of the nonlinear fitting algorithm, the uncertainty and the bias have been studied. A new method based on Box's estimation was developed to calculate the bias of estimated surface parameters, i.e., the 
radius. The method is significantly faster and more convenient than a computer simulation process.

\section{Methodology}

In this work, a theoretical study of an aspheric fitting algorithm is presented. A total or direct aspheric surface fitting algorithm is proposed. The "total" denotes that the estimation of all parameters will be performed at the same time. The fitting algorithm is based on the nonlinear least-squares sphere fitting algorithm [10], and uses a transformation of the aspheric surface equation to simplify the aspheric model.

The fitting process is applied to raster scan data [4], as used in the measurement of aspheric surfaces [11]. A square sampling strategy is used in the theoretical investigation where the result is expected to be tied with experimental work in the future. To simplify the theoretical study, the sampling interval $(\Delta l)$, the number of data points $(n)$ and the scanning length $(l)$ are defined to be the same in both the $\mathrm{X}$ and the $\mathrm{Y}$ axes.

The total aspheric surface fitting algorithm is tested on computer simulated ideal surfaces and surfaces superimposed with random irregularities. Both 2D aspheric curves and 3D aspheric surfaces are considered. Surface parameters and the standard deviation of residuals with the best-fit aspheric surface removed will be used to evaluate the "goodness" of surface fitting results. It is assumed that the centre of the aspheric surface is well aligned and normal to the sampling area. The positioning and surface alignment issues have been discussed in $[12,13]$ and will not be further discussed in this work. 


\section{Mathematic derivation}

The solution of a $6^{\text {th }}$ order aspheric function is illustrated in order to demonstrate the fitting approach. The least-squares algorithm aims to minimize the square of the residual $A \boldsymbol{x}-B$ in order to solve the system of linear equations.

$$
A x=B
$$

The example aspheric surface function is shown in Equation (3). Vectors $\{\boldsymbol{x}, \boldsymbol{y}, \boldsymbol{z}\}$ indicate the position of discrete surface points in a coordinate system. The four parameters $\left\{c, k, A_{4}, A_{6}\right\}$ to be estimated are the reciprocal of the vertex radius $(1 / \mathrm{R})$, the conic constant and the polynomial coefficients. The detail of these parameters can be found in Section 1.1.

$z=\frac{c \boldsymbol{r}^{2}}{1+\sqrt{1-(1+k) c^{2} \boldsymbol{r}^{2}}}+A_{4} \boldsymbol{r}^{4}+A_{6} \boldsymbol{r}^{6}$

Where $\quad r=\sqrt{x^{2}+y^{2}}$

The process consists of two steps. The first step is to estimate parameters by using the linear least-squares algorithm and the estimated values can be consequently used in the second step where a nonlinear leastsquares approach is used to improve the initial estimation. 


\subsection{The initial estimation of parameters}

Let $m=1+k$, such that Equation (3) is transferred into the form:

$$
c \boldsymbol{r}^{2}-2\left(z-A_{4} \boldsymbol{r}^{4}-A_{6} \boldsymbol{r}^{6}\right)+m c\left(z-A_{4} \boldsymbol{r}^{4}-A_{6} \boldsymbol{r}^{6}\right)^{2}=0
$$

Let $m c=s$ and eexpand Equation (4) as:

$$
z=\frac{1}{2} s z^{2}+\frac{1}{2} c \boldsymbol{r}^{2}+A_{4} \boldsymbol{r}^{4}-s A_{4} z \boldsymbol{r}^{4}+A_{6} \boldsymbol{r}^{6}-s A_{6} z \boldsymbol{r}^{6}+\frac{1}{2} s A_{4}^{2} \boldsymbol{r}^{8}+s A_{4} A_{6} \boldsymbol{r}^{10}+\frac{1}{2} s A_{6}^{2} \boldsymbol{r}^{12}
$$

If we let

$$
\begin{array}{lll}
p_{1}=\frac{s}{2}=\frac{m c}{2} & p_{2}=\frac{c}{2} & p_{3}=A_{4} \\
p_{4}=-s A_{4}=-A_{4} m c & p_{5}=A_{6} & p_{6}=-A_{6} s=-A_{6} m c \\
p_{7}=\frac{A_{4}^{2} s}{2}=\frac{A_{4}^{2} m c}{2} & p_{8}=-A_{4} A_{6} s=-A_{4} A_{6} m c & p_{9}=\frac{A_{6}^{2} s}{2}=\frac{A_{6}^{2} m c}{2}
\end{array}
$$

Then Equation (5) can be presented as:

$$
\boldsymbol{z}=p_{1} z^{2}+p_{2} \boldsymbol{r}^{2}+p_{3} \boldsymbol{r}^{4}+p_{4} z \boldsymbol{r}^{4}+p_{5} \boldsymbol{r}^{6}+p_{6} \boldsymbol{z} \boldsymbol{r}^{6}+p_{7} \boldsymbol{r}^{8}+p_{8} \boldsymbol{r}^{10}+p_{9} \boldsymbol{r}^{12}
$$


function of parameters $\left\{p_{1}, p_{2}, p_{3}, \ldots p_{9}\right\}$. Therefore, the linear least-squares algorithm can be applied to obtain the estimates of the parameters, where the linear least-squares method minimizes the summed square of the residuals. It is similar to the approach used by Forbes [10] for fitting spherical surfaces. More information regarding fitting complex functions can be found in [14-16].

Based on the linear least-squares algorithm, the parameters from Equation (6) can be solved in Equation (8).

$$
\left|\begin{array}{ccccc}
\sum \boldsymbol{a}_{1} \cdot \boldsymbol{a}_{1} & \sum \boldsymbol{a}_{1} \cdot \boldsymbol{a}_{2} & \sum \boldsymbol{a}_{1} \cdot \boldsymbol{a}_{3} & \cdots & \sum \boldsymbol{a}_{1} \cdot \boldsymbol{a}_{9} \\
\sum \boldsymbol{a}_{2} \cdot \boldsymbol{a}_{1} & \sum \boldsymbol{a}_{2} \cdot \boldsymbol{a}_{2} & \sum \boldsymbol{a}_{2} \cdot \boldsymbol{a}_{3} & \cdots & \sum \boldsymbol{a}_{2} \cdot \boldsymbol{a}_{9} \\
\sum \boldsymbol{a}_{3} \cdot \boldsymbol{a}_{1} & \sum \boldsymbol{a}_{3} \cdot \boldsymbol{a}_{2} & \sum \boldsymbol{a}_{3} \cdot \boldsymbol{a}_{3} & \cdots & \sum \boldsymbol{a}_{3} \cdot \boldsymbol{a}_{9} \\
\vdots & \vdots & \vdots & \ddots & \vdots \\
\sum \boldsymbol{a}_{9} \cdot \boldsymbol{a}_{1} & \sum \boldsymbol{a}_{9} \cdot \boldsymbol{a}_{2} & \sum \boldsymbol{a}_{9} \cdot \boldsymbol{a}_{3} & \cdots & \sum \boldsymbol{a}_{9} \cdot \boldsymbol{a}_{9}
\end{array}\right| \cdot\left|\begin{array}{c}
p_{1} \\
p_{2} \\
p_{3} \\
\vdots \\
p_{9}
\end{array}\right|=\left|\begin{array}{c}
\sum \boldsymbol{b}_{1} \cdot \boldsymbol{a}_{1} \\
\sum \boldsymbol{b}_{1} \cdot \boldsymbol{a}_{2} \\
\sum \boldsymbol{b}_{1} \cdot \boldsymbol{a}_{3} \\
\vdots \\
\sum \boldsymbol{b}_{1} \cdot \boldsymbol{a}_{9}
\end{array}\right|
$$

Where,

$$
\begin{array}{lllll}
a_{1}=z^{2} & a_{2}=r^{2} & a_{3}=r^{4} & a_{4}=z \cdot r^{4} & a_{5}=r^{6} \\
a_{6}=z \cdot r^{6} & a_{7}=r^{8} & a_{8}=r^{10} & a_{9}=r^{12} & b_{1}=2 z
\end{array}
$$

From Equation (8), the vector of parameters $\boldsymbol{p}=\left\{p_{1}, p_{2}, p_{3}, \ldots, p_{9}\right\}$ can be calculated. However, they are not the final parameters required. With the known value of the parameters $\left\{p_{1}, p_{2}, p_{3}, \ldots, p_{9}\right\}$, the value of parameters $\left\{c, m, A_{4}, A_{6}\right\}$ of the aspheric surface equation can be calculated with respect to the vector of parameters $\boldsymbol{p}$, using the relationships between parameters $\left\{p_{1}, p_{2}, p_{3}, \ldots, p_{9}\right\}$ and parameters $\left\{c, m, A_{4}, A_{6}\right\}$ as shown in Equation (6). 
There are nine equations for four parameters, where four equations are sufficient to solve a function with four parameters. Therefore, the selection of different equations may lead to different results in the initial estimation. However, as the values estimated from this stage are only used to start the iteration in the next step, the values do not represent the final estimation and the selection of equations will not be further discussed here.

The estimation of parameters can be calculated as shown in Equation (9), originate from Equation (6).

$$
c=2 p_{2} \quad m=\frac{p_{1}}{p_{2}} \quad A_{4}=p_{3} \quad A_{6}=p_{5}
$$

\subsection{The nonlinear least-squares estimation}

It is assumed that the aspheric surface algorithm has a root and has a small residual following the estimation of the starting values derived above, then the Gauss-Newton iteration approach is used to solve the non-linear equations, since the Gauss-Newton method converges quickly if the initial guess is close to the true value. For example, parameters with the values $\{1,1,1,1\}$ may be sufficient to start iteration; however, the convergence may take more steps than the iteration with a good initial estimation; and with the start values far from the expected value, the iteration may not converge.

With the initial estimation from the linear least-squares technique, the approximation of the true value of the parameters is calculated by using the Gauss-Newton iteration approach. Additional detail of this approach are 
detailed in [17]. In summary the process minimizes the difference between the observed data $z$ and the

estimated data $\widehat{z}$ based on the parameters estimated from the linear least-squares process.

$d=\widehat{z}-z$

The iteration procedure is described as below:

a. Use the linear least-squares algorithm to obtain initial estimation of $c, m, A_{4}$ and $A_{6}$, refer to

Section 3.1.

b. Solve the non-linear least-squares system

$$
\boldsymbol{J}\left[\begin{array}{c}
\Delta c \\
\Delta p \\
\Delta A_{4} \\
\Delta A_{6}
\end{array}\right]=-\boldsymbol{d}
$$

The corresponding Jacobian matrix is:

$$
\boldsymbol{J}=\left[\begin{array}{llll}
\frac{\partial \boldsymbol{d}}{\partial c} & \frac{\partial \boldsymbol{d}}{\partial m} & \frac{\partial \boldsymbol{d}}{\partial A_{4}} & \frac{\partial \boldsymbol{d}}{\partial A_{6}}
\end{array}\right]^{T}
$$

Where the first partial derivatives of $\boldsymbol{d}$ are: 


$$
\begin{aligned}
& \frac{\partial \boldsymbol{d}}{\partial c}=\frac{\boldsymbol{r}^{2}}{\sqrt{1-m c^{2} \boldsymbol{r}^{2}}}-\frac{1}{m c^{2}}+\frac{\sqrt{1-m c^{2} \boldsymbol{r}^{2}}}{m c^{2}} \\
& \frac{\partial \boldsymbol{d}}{\partial m}=\frac{c^{2} \boldsymbol{r}^{2}}{2 m c \sqrt{1-m c^{2} \boldsymbol{r}^{2}}}-\frac{1-\sqrt{1-m c^{2} \boldsymbol{r}^{2}}}{m^{2} c} \\
& \frac{\partial \boldsymbol{d}}{\partial A_{4}}=\boldsymbol{r}^{4} \\
& \frac{\partial \boldsymbol{d}}{\partial A_{6}}=\boldsymbol{r}^{6}
\end{aligned}
$$$$
\left|\begin{array}{cccc}
\sum \frac{\partial \boldsymbol{d}}{\partial c} \cdot \frac{\partial \boldsymbol{d}}{\partial c} & \sum \frac{\partial \boldsymbol{d}}{\partial c} \cdot \frac{\partial \boldsymbol{d}}{\partial m} & \sum \frac{\partial \boldsymbol{d}}{\partial c} \cdot \frac{\partial \boldsymbol{d}}{\partial A_{4}} & \sum \frac{\partial \boldsymbol{d}}{\partial c} \cdot \frac{\partial \boldsymbol{d}}{\partial A_{6}} \\
\sum \frac{\partial \boldsymbol{d}}{\partial m} \cdot \frac{\partial \boldsymbol{d}}{\partial c} & \sum \frac{\partial \boldsymbol{d}}{\partial m} \cdot \frac{\partial \boldsymbol{d}}{\partial m} & \sum \frac{\partial \boldsymbol{d}}{\partial m} \cdot \frac{\partial \boldsymbol{d}}{\partial A_{4}} & \sum \frac{\partial \boldsymbol{d}}{\partial m} \cdot \frac{\partial \boldsymbol{d}}{\partial A_{6}} \\
\sum \frac{\partial \boldsymbol{d}}{\partial A_{4}} \cdot \frac{\partial \boldsymbol{d}}{\partial c} & \sum \frac{\partial \boldsymbol{d}}{\partial A_{4}} \cdot \frac{\partial \boldsymbol{d}}{\partial m} & \sum \frac{\partial \boldsymbol{d}}{\partial A_{4}} \cdot \frac{\partial \boldsymbol{d}}{\partial A_{4}} & \sum \frac{\partial \boldsymbol{d}}{\partial A_{4}} \cdot \frac{\partial \boldsymbol{d}}{\partial A_{6}} \\
\sum \frac{\partial \boldsymbol{d}}{\partial A_{6}} \cdot \frac{\partial \boldsymbol{d}}{\partial c} & \sum \frac{\partial \boldsymbol{d}}{\partial A_{6}} \cdot \frac{\partial \boldsymbol{d}}{\partial m} & \sum \frac{\partial \boldsymbol{d}}{\partial A_{6}} \cdot \frac{\partial \boldsymbol{d}}{\partial A_{4}} & \sum \frac{\partial \boldsymbol{d}}{\partial A_{6}} \cdot \frac{\partial \boldsymbol{d}}{\partial A_{6}}
\end{array}\right|=-\left|\begin{array}{c}
\sum \frac{\partial \boldsymbol{d}}{\partial c} \cdot \boldsymbol{d} \\
\Delta \frac{\partial \boldsymbol{d}}{\Delta A_{4}} \cdot \boldsymbol{d} \\
\Delta A_{6}
\end{array}\right|=\left|\begin{array}{c}
\frac{\partial \boldsymbol{d}}{\partial A_{4}} \cdot \boldsymbol{d} \\
\sum \frac{\partial \boldsymbol{d}}{\partial A_{6}} \cdot \boldsymbol{d}
\end{array}\right|
$$

c. Update the parameter estimates

$$
\begin{aligned}
& c:=c+\Delta c \\
& m:=m+\Delta m \\
& A_{4}:=A_{4}+\Delta A_{4} \\
& A_{6}:=A_{6}+\Delta A_{6}
\end{aligned}
$$

d. The iteration stops only if the convergence condition is satisfied, otherwise go back to step b. 
For a 3D system, the difference is the three-axis data involved.

$$
\boldsymbol{r}=\sqrt{\left(\boldsymbol{x}-x_{0}\right)^{2}+\left(\boldsymbol{y}-y_{0}\right)^{2}}
$$

where $\left(x_{0}, y_{0}\right)$ is the location of the vertex point of the surface. In the following tests, it has been assumed that the vertex points of aspheric surfaces are all located at the origin. Therefore, $x_{0}$ and $y_{0}$ are both equal to zero.

\subsection{Additional condition}

There is a radical expression, $\sqrt{1-(1+k) c^{2} r^{2}}$, in Equation (3). Therefore, the value of parameter $k$ must satisfy the Inequality (16) in order to keep values of $z$ real numbers.

$k<\frac{1}{c^{2} r_{\max }^{2}}-1$

Where

$r_{\max }=\sqrt{\left(\boldsymbol{x}-x_{0}\right)_{\max }^{2}+\left(\boldsymbol{y}-y_{0}\right)_{\max }^{2}}$ 
This issue occurs in the iteration procedure. In some cases, the aspheric fitting algorithm can not succeed in estimating the parameters if the initial estimation exceeds this condition. Therefore, the value of parameter $k$ has to be reset. The issue will be further clarified in the following section.

\subsection{Transformation of surfaces with negative vertex radius}

Aspheric surfaces can be concave, convex or planar. In optical systems it is common to see the designed vertex radius of one side of the aspheric surface with a positive value and the other side negative. For such an aspheric lens it is common that the conic section of one surface side is a prolate ellipsoid and the other side is a hyperboloid.

In the measurement and fitting process, to unify the problem, for an unknown measured surface, it is convenient to assume that the vertex radius is positive. Therefore, in order to compare the measured surface with a designed surface, the surface Equation (3) with a negative vertex radius will be transformed into a positive form, as shown in Equation (17).

$$
z^{\prime}=\frac{c^{\prime} \boldsymbol{r}^{2}}{1+\sqrt{1-\left(1+k^{\prime}\right) c^{\prime 2} \boldsymbol{r}^{2}}}+A_{4}^{\prime} \boldsymbol{r}^{4}+A_{6}^{\prime} \boldsymbol{r}^{6}=-\boldsymbol{z}
$$

where

$$
c^{\prime}=-c \quad k^{\prime}=k \quad A_{4}^{\prime}=-A_{4} \quad A_{6}^{\prime}=-A_{6}
$$




\subsection{Quality of fitting}

A question that must be addressed is how well the algorithm fits the data. Parameters are common references in the evaluation of a fitting process. The difference between the best-fit parameters and the designed parameters describes how close the fitting result is to the designed values. However, the estimation of parameters may not be able to reveal the quality of fitting if the design model of the measured surface is unknown. For example, a near planar surface can be fitted by a spherical surface or plane surface model, where the fitting results may converge in both cases. The information extracted from residuals, i.e., the difference between the measured data and the best-fit data, can provide more information in order to reveal the quality of fitting. In practise, however, the magnitude of the surface residuals with the best-fit surface removed does not only depend on the goodness of the fitting algorithm, but also the quality of the measured surfaces, i.e., the magnitude of the surface roughness. Thus, observation of the surface residuals with the bestfit surface removed is not enough to judge the goodness of a fit. In this work, both the best-fit parameters and the residuals with the best-fit surface removed will be considered to give a complete view of test results.

\section{Algorithm testing}

The algorithm has been applied to a selection of both computer simulated 2D curves and 3D surfaces, with and without random irregularities. Surfaces with different orders of polynomials are also tested.

There are some general terms used in the test: 
LLS: linear least-squares

NLLS: nonlinear least-squares

Table 1 shows the testing sequence. There are eight studied. For Cases 1 to 6 , the aspheric fitting algorithm has been tested on computer simulated 2D aspheric curves (Cases 1-6) and 3D aspheric surfaces (Cases 7-8).

There are more 2D cases presented than 3D as the results are more easily viewed. The results for the 8 case studies are shown in Tables 2- 9 and Figure's 3-10. In each case, both the linear least-squares and nonlinear least-squares fitting results are compared with the designed parameters. The sum of the absolute change of

parameters, ( $\boldsymbol{\delta}=\sum\left|\Delta \boldsymbol{p}_{s}\right|$ ) for each iteration is recorded and the residuals of the investigated curve/surface with best-fit curve/surface removed displayed.

\subsection{Tests on simulated ideal aspheric curves/surfaces (Cases 1-5 and Case 7)}

The estimations of vertex radii using the linear least-squares technique is generally close to the designed values, for example in Table 2, the LLS result for the vertex radius is within 200nm of the designed value.. The nonlinear process further improves these initial fitting results.

\section{- Parameters}

The results of the parameter fits from the first stage of the fitting process using the LLS method are shown for Case 1 in Table 2 . As shown the radius error is $200 \mathrm{~nm}(0.0004 \%)$, the conic constant error is approximately 
$10 \%$, while the $\mathrm{A}_{6}$ error is very large. The errors in the parameters following the NLLS fit are then negligible as shown in Table 2, and in the residual graph in Figure 3. In the figure the first curve is the ideal surface, the middle curve shows the convergence of the parameters with iterations and the final graph on the right is the residual error.

In the study of Case 2 (corresponding to Figure 4 and Table 3), the conic constant error after the LLS fit is very large. This has a direct influence on the quality of the fit following the NLLS process, however the data shown in Figure 4 shows convergence after 7 iterations and a residual surface error comparable to the data in Figure 3. Although the initial estimation of the vertex radius using the linear least-squares LLS algorithm is $0.07 \mathrm{~mm}$ different from the designed value, the estimation has not been further improved by using the nonlinear process. The case shows a non-unique solution to the fitting problem. This problem may arise from the convergence of the Gauss-Newton iteration process. Although the Gauss-Newton iteration process converges rapidly, the iteration can get stuck in local minima [18], particularly for large fits with many parameters. Thus, the best-fit parameters estimated by the Gauss-Newton iteration algorithm may not be the best-fit results satisfying a global minimum. Many techniques have been proposed for the global optimisation contain random processes [19]. For example, the multistart technique searches for the global minimum by varying the starting points of the iteration. Although this method is a technique frequently used, the efficiency of the search routine is extremely low.

In the remaining tests on two dimensional ideal data sets (Cases 3, 4 and 5), aspheric curves with different 
orders of polynomials, from 6th to 12th order, are simulated. In all cases the measurement results are close to the designed parameters. In the study of Case 4 the significant error in the conic constant term and the polynomial terms after the LLS fitting process results in a poor convergence of the NLLS fit; sometimes the iteration process even diverges. However the resultant residuals of the surface fit are still at a very low value $\left(\mathrm{x} 10^{-15} \mathrm{~mm}\right)$.

In the cases of the 3D surface the fitting algorithm produces identical results compared with designed parameters shown in Case 7 (refer to Figure 9 and Table 8). This is the same design equation as that used in Case 1 above.

\section{- Iteration procedure}

The Gauss-Newton iteration process produces fast convergence. Most of the tests converge within 10 steps except Case 4 (see Figure 6 and Table 5). In that case, it can be found that the rate of convergence of the parameter to the designed value was rapid at the beginning of the iterations, but this declined and convergence was achieved slowly towards the end. This may due to the convergence property of the Gauss-Newton iteration process, where the method may not converge when the iteration starts far from the solution.

\section{- Residuals}


The sizes of residuals for all the tests on computer simulated ideal curves/ surfaces are very small. The mean

value of the residuals is in the order of $10^{-15}$, equivalent to numerical precision. The residuals at the centre of the form are small and the values increase towards the edge of the form. However, there is an exceptionCase 4 (see Figure 6 and Table 5).

Daniel and Wood suggested that the pattern of residuals are related to components of the equation [17].

However, the pattern in Case 4 does not show a significant relationship between the residuals and the value of $r$. Besides, the values of the residuals are extremely small. All of these suggest that the residuals might be caused by the poor convergence. The idea has been further tested by increasing the iteration condition to $10^{-10}$. The iteration took more steps (581) to fulfil the convergence condition. However the size of residuals is similar as other test results.

\subsection{Tests on simulated aspheric curves/surfaces with random irregularities}

Figure 8 and Table 7 show Case 6 for the 2D data and Figure 10 and Table 9 show Case 8 for the 3D data with same surface designed parameters. The standard deviation of surface irregularities for both cases is $50 \mathrm{~nm}$. In Case 6, with the best-fit curve removed, the standard deviation of the residuals is $49.9 \mathrm{~nm}$, which is only 0.1 nm different from the simulated value (refer to Figure 8 and Table 7). The corresponding result in fitting a computer simulated aspheric surface (Case 8) is even better (refer to Figure 10 and Table 9), the difference of the standard deviation between fitted results and the designed value is only $0.0009 \mathrm{~nm}$. 


\section{Conclusion and discussion}

In this paper, a new aspheric surface fitting algorithm has been developed. This method is superior to previous attempts described by other researchers $[7,13,20,21]$ because it estimates all parameters and it offers greater flexibility than other methods with less steps required. The algorithm utilises the nonlinear least-squares technique.

The fitting algorithm has been tested on both 2D and 3D data sets. Both ideal surfaces and surfaces with irregularities have been considered. The algorithm produces reliable fitting results compared with designed values in most of the cases presented. The fitting process converges very rapidly on both simulated ideal curves/surfaces and curve/surface superimposed with random irregularities.

Issues regarding the application of this method have been discussed. The main problem is the stability of the fitting algorithm. Gauss-Newton iteration has been used to find the best-fit parameters. The iteration method does not always lead to convergence of the parameters, which can result in errors in the estimations. This issue needs further investigation before the algorithm can be applied in fitting measured aspheric surfaces. 
[1] Douthwaite WA. Contact lens optics and lens design. Butterworth-Heinemann Ltd., 1995

[2] Heynacher E. Aspheric optics-How they are made and why they are needed. Physics in Technology $1979 ; 10 ; 124-31$

[3] Mcister D. Aspheric lenses: Optics and applications. Lens Talk 1998;26(25)

[4] Stout KJ. Development of methods for the characterisation of roughness in three dimensions. UK: Penton Press, 2000

[5] Aceves-Campos H. Profile identification of aspheric lenses Applied Optics 1998;37(34); 8149-50

[6] Zhang Z. Parameter estimation techniques: a tutorial with application to conic fitting. Image and Vision Computing 1997;15(1); 59-76

[7] Gugsa S and Davies A. Monte Carlo analysis for the determination of the conic constant of an aspheric micro lens based on a scanning white light interferometric measurement. In: Hatheway Alson E. editors:

Proceedings of the SPIE. 2005, 2005. 92-102

[8] Sun W. Precision measurement and characterisation of spherical and aspheric surfaces. 2007.University of Southampton. PhD Thesis. 149

[9] Sun W, Hill M and McBride JW. An investigation of the robustness of the nonlinear least-squares sphere fitting method to small segment angle surfaces. Precision Engineering 2008;32(1); 55-62

[10] Forbes AB. Robust circle and sphere fitting by least squares. 1989.(DITC 153/89).NPL

[11] Difference AMtEt, and BCiR, After COSP and Keratomileusis Lis.

[12] Hill M, Jung M and McBride JW. Separation of form from orientation in 3D measurements of aspheric surfaces with no datum. International Journal of Machine Tools \& Manufacture 2002;42; 457-66

[13] Jung M. Assessment of curved, rotationally symmetric surfaces in three dimensions. 1999.University of Southampton. Ph.D Thesis. 131

[14] Jaccard J, Turrisi R and Wan CK. Interaction effects in multiple regression. Sage Publications, Inc., 1990

[15] Berry WD and Feldman S. Multiple regression in practice. Sage Publications, Inc., 1985

[16] Jaccard J and Wan CK. Lisrel approaches to interaction effects in multiple regression. Sage Publications, Inc., 1996

[17] Daniel C and Wood FS. Fitting equations to data:computer analysis of multifactor data. John Wiley\&Sons, Inc, 1980

[18] Woodford CH and Phillips C. Numerical methods with worked examples. 1997

[19] Towards global optimisation. North-Holland Publishing Company, 1975

[20] Stout KJ, et al. The development of methods for the characterisation of roughness in three dimensions. Butterworth-Heinemann, 1993

[21] Sourlier D and Bucher A. Surface-independent, theoretically exact bestfit for arbitrary sculptured, complex, or standard geometries. Precision Engineering 1995;17(2); 101-13 


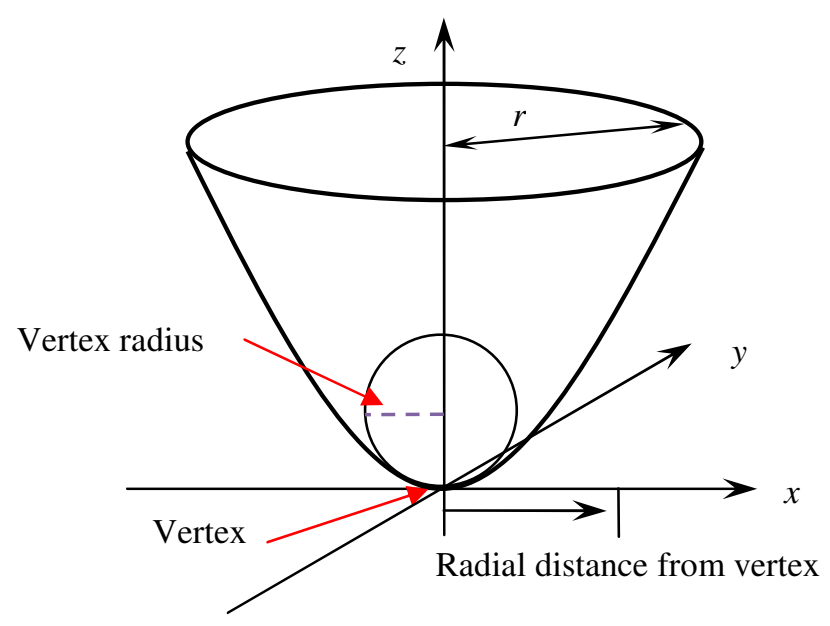

Figure 1. Schematic of an aspheric surface 


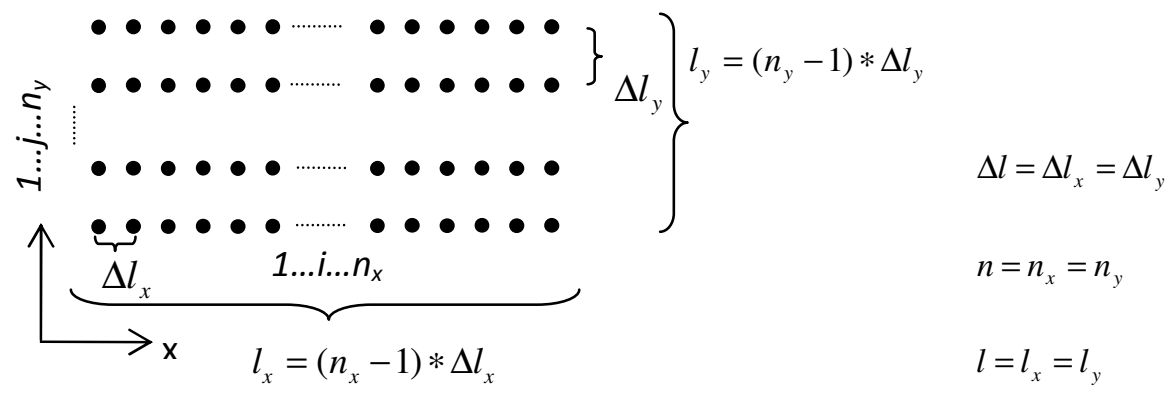

Figure 2. Notation of sampling area 

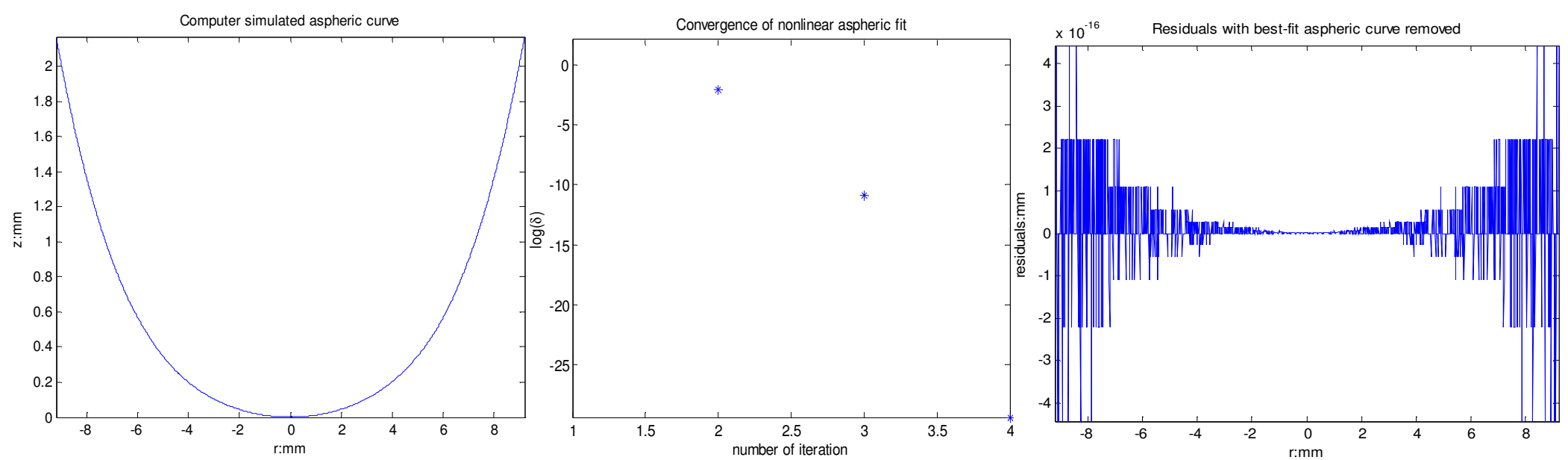

Figure 3 Case 1: Fitting results of a 6 th order ideal aspheric curve (2D).

(Left): Simulated 6th order aspheric curve. (Middle): The convergence of parameters-log $(\delta)$ in each iteration step. (Right): Residuals with the best-fit 6th order curve removed. 

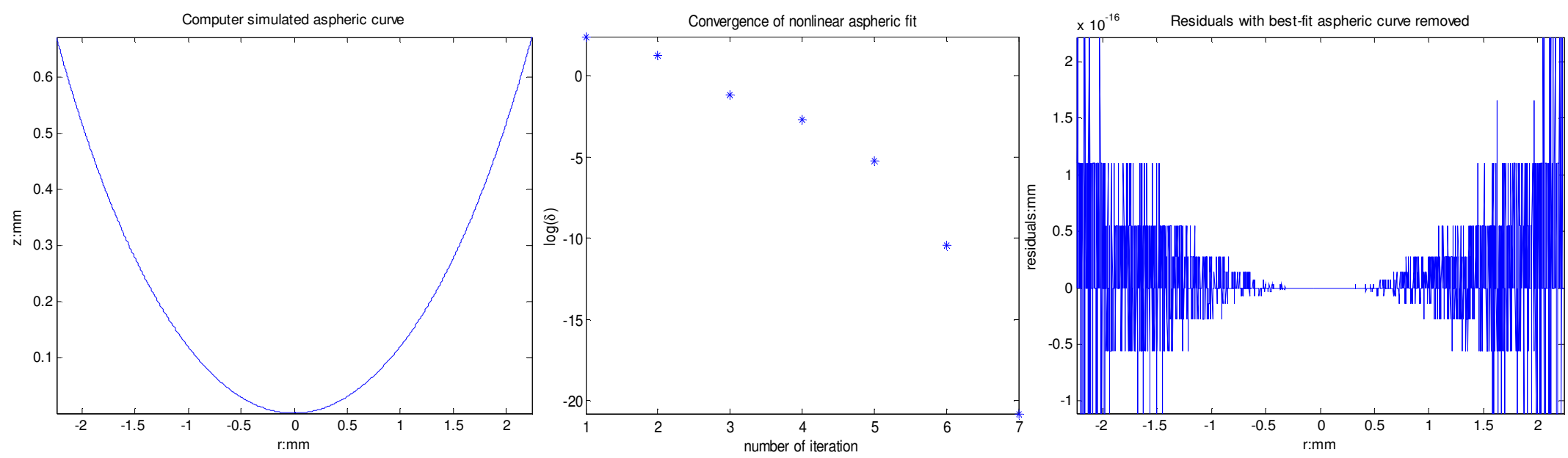

Figure 4 Case 2: Fitting results of a 6th order ideal aspheric curve.

(Left): Simulated 6th order aspheric curve. (Middle): The convergence of parameters-log $(\delta)$ in each iteration step. (Right): Residuals with the best-fit 6th order curve removed. 

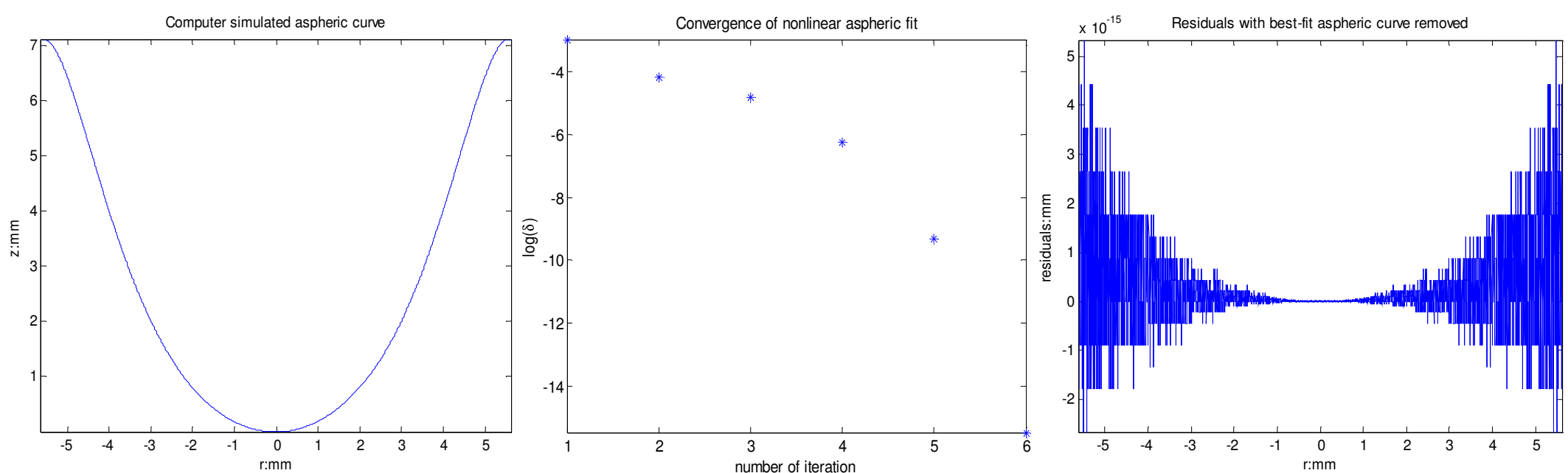

Figure 5 Case 3: Fitting results of an 8th order ideal aspheric curve.

(Left): Simulated 8th order aspheric curve. (Middle): The convergence of parameters-log $(\delta)$ in each iteration step. (Right): Residuals with the best-fit 8th order curve removed. 

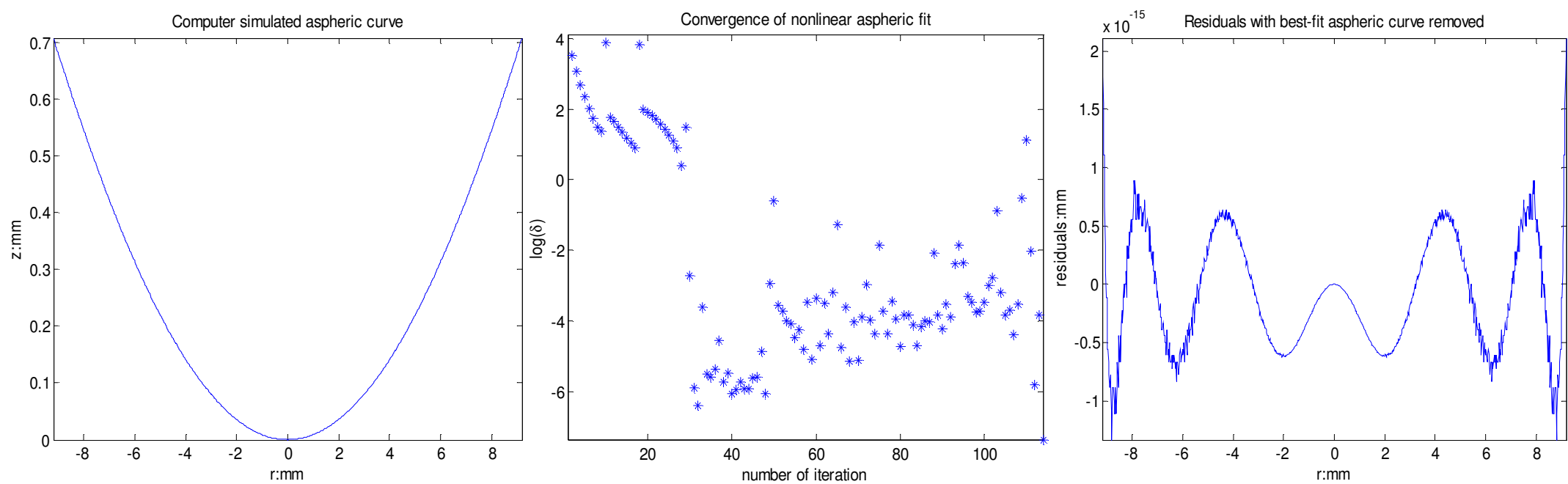

Figure 6 Case 4: Fitting results of a 10th order ideal aspheric curve.

(Left): Simulated 10th order aspheric curve. (Middle): The convergence of parameters—log $(\delta)$ in each iteration step. (Right): Residuals with the best-fit 10th order curve removed. 

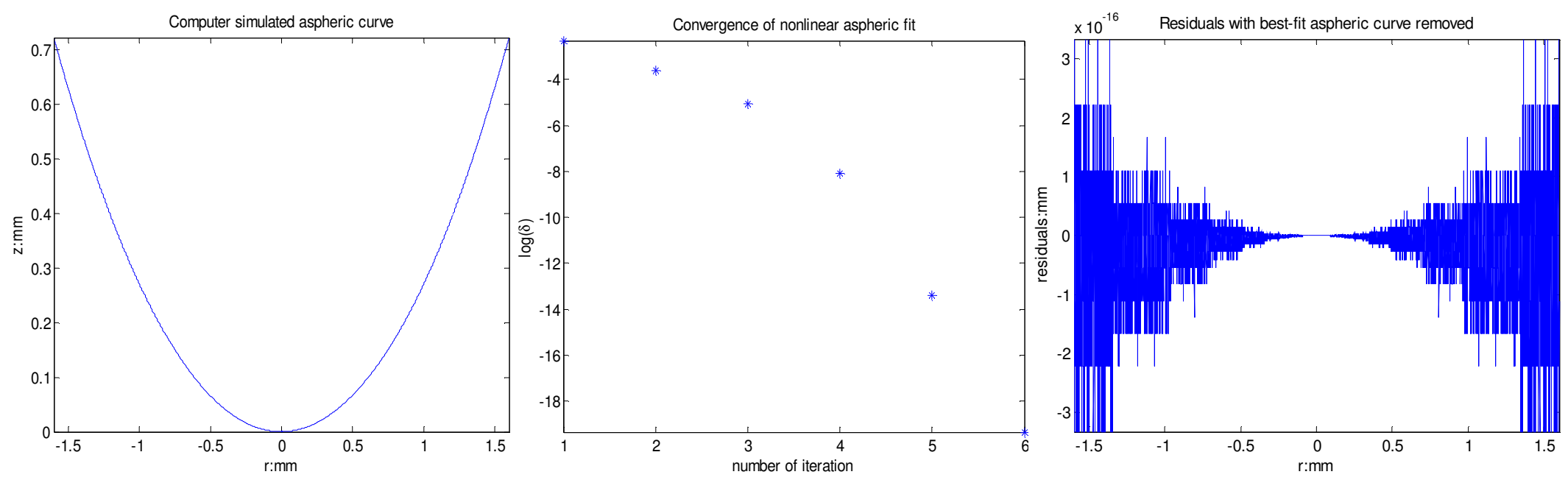

Figure 7 Case 5: Fitting results of a 12th order ideal aspheric curve.

(Left): Simulated 12th order aspheric curve. (Middle): The convergence of parameters-log $(\delta)$ in each iteration step. (Right): Residuals with the best-fit 12th order curve removed. 

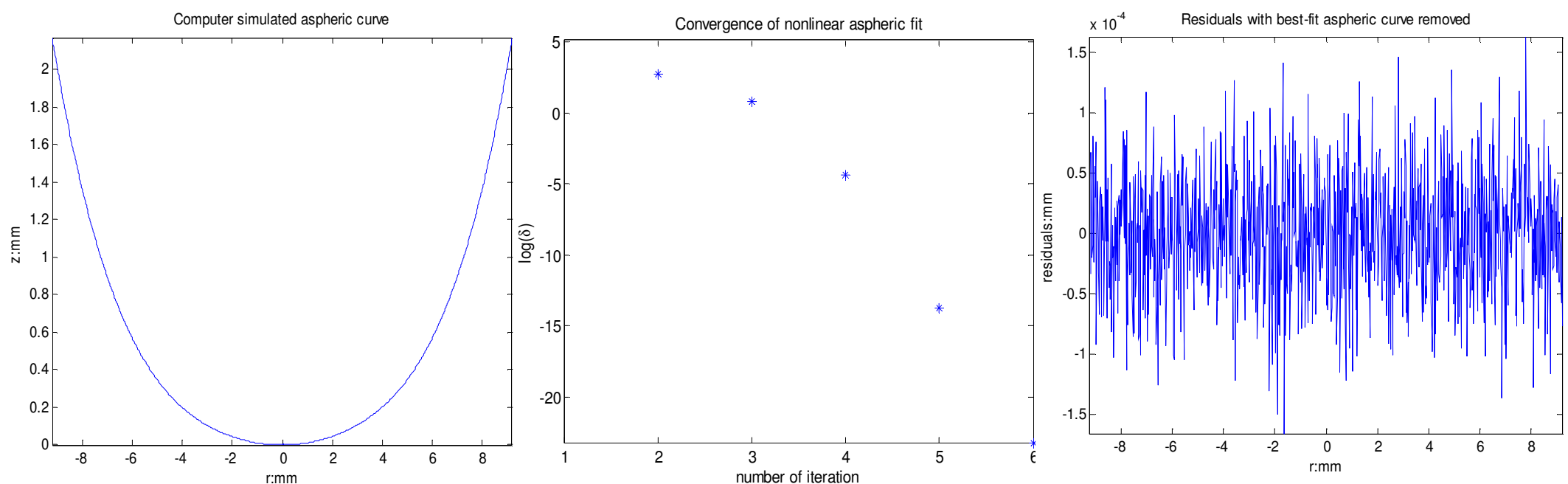

Figure 8 Case 6: Fitting results of a 6th order aspheric curve superimposed with irregularities.

(Left): Simulated 6th order aspheric curve. (Middle): The convergence of parameters-log $(\delta)$ in each iteration step. (Right): Residuals with the best-fit 6th order curve removed. 

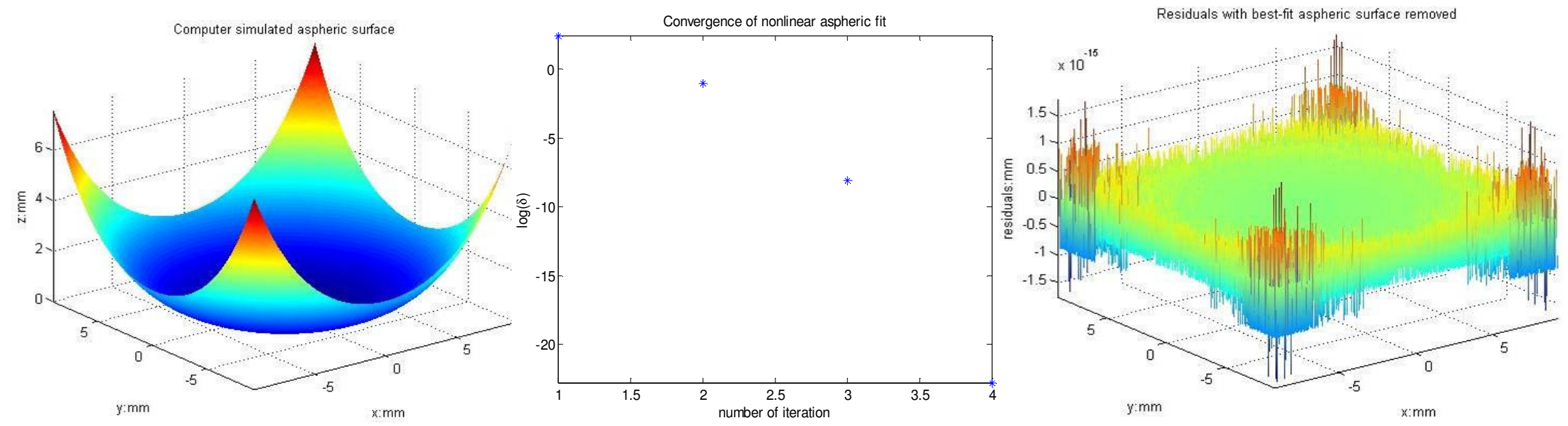

Figure 9 Case 7: Fitting results of a 6th order aspheric surface.

(Left): Simulated 6th order aspheric surface. (Middle): The convergence of parameters-log $(\delta)$ in each iteration step. (Right): Residuals with the best-fit 6th order surface removed. 

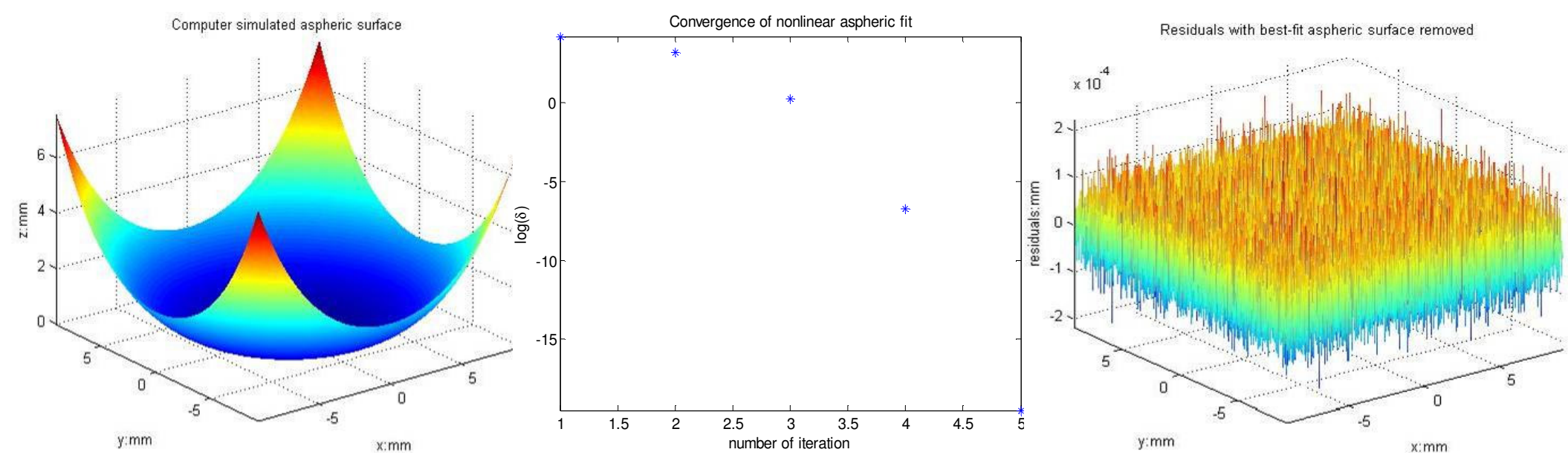

Figure 10 Case 8: Fitting results of a 6th order aspheric surface superimposed with surface noise.

(Left): Simulated 6th order aspheric surface. (Middle): The convergence of parameters-log( $\delta$ ) in each iteration step. (Right): Residuals with the best-fit 6th order surface removed. 


\begin{tabular}{|c|c|c|c|c|}
\hline & Dimension & Case & Order & Surface irregularity \\
\hline \multirow{8}{*}{$\mathrm{CS}$} & \multirow{6}{*}{$2 \mathrm{D}$} & Case 1 & 6th & None \\
\hline & & Case 2 & 6th & None \\
\hline & & Case 3 & 8 th & None \\
\hline & & Case 4 & 10th & None \\
\hline & & Case 5 & 12th & None \\
\hline & & Case 6 & 6th & $50 \mathrm{~nm}$ \\
\hline & \multirow{2}{*}{$3 \mathrm{D}$} & Case 7 & 6th & None \\
\hline & & Case 8 & 6th & $50 \mathrm{~nm}$ \\
\hline
\end{tabular}

Table 1. The test sequence of the algorithm.

'CS' stands for the computer simulated data. 


\begin{tabular}{ccccc}
\hline Iteration:4 & $R$ & $k$ & $A_{4}$ & $A_{6}$ \\
\hline Designed & 44.577884 & $-1.710312 \mathrm{e}+2$ & $2.316294 \mathrm{e}-4$ & $3.495852 \mathrm{e}-8$ \\
\hline LLS results & 44.577696 & $-1.888241 \mathrm{e}+2$ & $2.565880 \mathrm{e}-4$ & $3.655224 \mathrm{e}-6$ \\
\hline NLLS results & 44.577884 & $-1.710312 \mathrm{e}+2$ & $2.316294 \mathrm{e}-4$ & $3.495852 \mathrm{e}-8$ \\
\hline
\end{tabular}

Table 2 Case 1: Fitting results of a 6th order ideal aspheric curve 


\begin{tabular}{ccccc}
\hline Iterations:7 & $R$ & $k$ & $A_{4}$ & $A_{6}$ \\
\hline Designed & 4.25 & -0.863601 & $1.77613 \mathrm{e}-4$ & $-1.55395 \mathrm{e}-5$ \\
\hline LLS results & 4.32 & 14.021781 & $2.29104 \mathrm{e}-4$ & $-3.63173 \mathrm{e}-3$ \\
\hline NLLS results & 4.32 & 0.648067 & $1.658530 \mathrm{e}-4$ & $-8.28138 \mathrm{e}-6$ \\
\hline Table 3 Case 2: Fitting results of a 6th order ideal aspheric curve
\end{tabular}




\begin{tabular}{cccccc}
\hline Iterations: 6 & $R$ & $k$ & $A_{4}$ & $A_{6}$ & $A_{8}$ \\
& & & & & \\
\hline Designed & 2.708638 & -0.8968698 & $2.788402 \mathrm{e}-03$ & $1.553377 \mathrm{e}-04$ & $-7.281244 \mathrm{e}-06$ \\
& & & & & \\
\hline LLS results & 2.708646 & -0.8154612 & $2.276917 \mathrm{e}-03$ & $1.802417 \mathrm{e}-03$ & $2.287627 \mathrm{e}-05$ \\
& & & & & \\
\hline NLLS results & 2.708638 & -0.8968698 & $2.788402 \mathrm{e}-03$ & $1.553377 \mathrm{e}-004$ & $-7.281244 \mathrm{e}-06$ \\
& & & & & \\
\end{tabular}

Table 4 Case 3: Fitting results of an 8th order ideal aspheric curve 


\begin{tabular}{ccccccc}
\hline Iterations: 141 & $R$ & $k$ & $A_{4}$ & $A_{6}$ & $A_{8}$ & $A_{10}$ \\
& & & & & & $-1.23 \mathrm{e}-14$ \\
\hline Designed & 56.031 & -3 & $-4.33 \mathrm{e}-06$ & $-9.76 \mathrm{e}-09$ & $-1.09 \mathrm{e}-12$ & \\
\hline LLS results & 56.0312 & -8.521368 & $3.21398 \mathrm{e}-04$ & $-8.5214 \mathrm{e}-07$ & $5.78769 \mathrm{e}-06$ & $-8.13332 \mathrm{e}-10$ \\
\hline NLS results & 56.031 & -3.00005 & $-4.32996 \mathrm{e}-06$ & $-9.7600 \mathrm{e}-09$ & $-1.08999 \mathrm{e}-12$ & $-1.2300 \mathrm{e}-14$ \\
\hline \multicolumn{5}{c}{ Table 5 Case 4. Fitting results of a 10th order ideal aspheric curve } & \\
\hline
\end{tabular}

Table 5 Case 4: Fitting results of a 10th order ideal aspheric curve 


\begin{tabular}{ccccccccc}
\hline Iterations: 6 & $R$ & $k$ & $A_{4}$ & $A_{6}$ & $A_{8}$ & $A_{10}$ & $A_{12}$ \\
\hline Designed & 1.898836 & -0.5603343 & $-6.8505495 \mathrm{e}-4$ & $-4.1501354 \mathrm{e}-4$ & $-4.4705513 \mathrm{e}-5$ & $-1.8065968 \mathrm{e}-5$ & $-2.1569936 \mathrm{e}-7$ \\
\hline LLS results & 1.898830 & -0.5846901 & $-2.0012490 \mathrm{e}-2$ & $1.352406670 \mathrm{e}-2$ & $-2.8030138 \mathrm{e}-3$ & $3.0542563 \mathrm{e}-3$ & $-4.7906130 \mathrm{e}-3$ \\
\hline NLLS results & 1.898836 & -0.5603343 & $-6.8505495 \mathrm{e}-4$ & $-4.1501354 \mathrm{e}-4$ & $-4.4705513 \mathrm{e}-5$ & $-1.8065968 \mathrm{e}-5$ & $-2.1569936 \mathrm{e}-7$ \\
\hline
\end{tabular}

Table 6 Case 5: Fitting results of a 12th order ideal aspheric curve 


\begin{tabular}{cccccc}
\hline Iterations: 6 & $R$ & $k$ & $A_{4}$ & $A_{6}$ & Noise(std) \\
\hline Designed & 44.577884 & $-1.710312 \mathrm{e}+2$ & $2.316294 \mathrm{e}-4$ & $3.495852 \mathrm{e}-8$ & $50 \mathrm{~nm}$ \\
\hline LLS results & 44.671917 & 7.932042 & $-9.766368 \mathrm{e}-6$ & $-5.391427 \mathrm{e}-6$ & $1.14 \mathrm{e}+6 \mathrm{~nm}$ \\
\hline NLLS results & 44.570579 & $-1.711257 \mathrm{e}+2$ & $2.316411 \mathrm{e}-4$ & $3.492993 \mathrm{e}-8$ & $49.9 \mathrm{~nm}$
\end{tabular}

Table 7 Case 6: Fitting results of a 6th order aspheric curve superimposed with random irregularities 


\begin{tabular}{ccccc}
\hline Iteration: 4 & $R$ & $k$ & $A_{4}$ & $A_{6}$ \\
& & & & \\
\hline Designed & 44.577884 & $-1.710312 \mathrm{e}+2$ & $2.316294 \mathrm{e}-4$ & $3.495852 \mathrm{e}-8$ \\
\hline LLS results & 44.571832 & $-1.823692 \mathrm{e}+2$ & $2.470864 \mathrm{e}-4$ & $1.416801 \mathrm{e}-6$ \\
\hline NLLS results & 44.577884 & $-1.710312 \mathrm{e}+2$ & $2.316294 \mathrm{e}-4$ & $3.495852 \mathrm{e}-8$ \\
\hline
\end{tabular}

Table 8 Case 7: Fitting results of a 6th order ideal aspheric surface 


\begin{tabular}{cccccc}
\hline Iteration: 5 & $R$ & $k$ & $A_{4}$ & $A_{6}$ & Noise $($ std $)$ \\
\hline Designed & 44.577884 & $-1.710312 \mathrm{e}+2$ & $2.316294 \mathrm{e}-4$ & $3.495852 \mathrm{e}-8$ & $50 \mathrm{~nm}$ \\
\hline LLS results & 44.835505 & $-0.782601 \mathrm{e}+2$ & $1.176835 \mathrm{e}-4$ & $-1.805674 \mathrm{e}-6$ & $1.695431 \mathrm{~mm}$ \\
\hline NLLS results & 44.578034 & $-1.710267 \mathrm{e}+2$ & $2.316284 \mathrm{e}-4$ & $3.496336 \mathrm{e}-8$ & $49.9991 \mathrm{~nm}$ \\
\hline
\end{tabular}

Table 9 Case 8: Fitting results of a 6th order aspheric surface superimposed with surface noise. 